participant education, HIV status, STI history, pre-sex alcohol use, sexual role, and partner sexual role).

Results 138 TW (median age 27, IQR: 22-33) provided data on their last sexual encounter with 376 distinct partners $(78 \%$ reporting 3 partners; $16 \%$ reporting 2; $6 \%$ reporting 1 ). Most (65.2\%) TW practiced receptive AI exclusively, 3.6\% practiced insertive AI exclusively, and $31.2 \%$ practiced both. I-CAI was more prevalent than R-CAI among TW with alcohol use disorders, drug use before sex, sexual role strain (performing a different role than preferred), and less than secondary education. ICAI was more prevalent with transactional sex partners $\left(\mathrm{PR}_{\mathrm{adj}}\right.$ : $1.54 ; \mathrm{p}=0.19$; reference: primary partners) and casual partners $\left(\mathrm{PR}_{\mathrm{adj}}: 1.39 ; \mathrm{p}=0.31\right)$, while R-CAI was more common with stable compared with transactional $\left(\mathrm{PR}_{\mathrm{adj}}: 0.67, \mathrm{p}=0.06\right)$ and casual partners $\left(\mathrm{PR}_{\mathrm{adj}}\right.$ : $\left.0.70, \mathrm{p}=0.14\right)$. I-CAI was significantly lower among HIV-positive TW $\left(\mathrm{PR}_{\mathrm{adj}}\right.$ : $0.53 ; \mathrm{p}=0.02$; reference: HIV-negative), while R-CAI was significantly higher among TW with unknown HIV status $\left(\mathrm{PR}_{\mathrm{adj}}: 1.91 ; \mathrm{p}=0.048\right)$.

Conclusion Examining partner-level I-CAI/R-CAI separately reveals different HIV/STI risk contexts/behaviours among TW. Future studies should explore implications of partner and AIassociated risk behaviours, including experience of sexual role strain and differences in TW's partner-specific perceptions of HIV/STI risk, sexual intimacy, gender affirmation, transactional sex demands, and condom use agency.

Disclosure of interest statement None.

\section{P04.28 EXPLORING CONTEXTUAL DIFFERENCES FOR RECEPTIVE AND INSERTIVE ROLE STRAIN AMONG TRANSGENDER WOMEN AND MEN WHO HAVE SEX WITH MEN IN LIMA, PERU}

${ }^{1}$ MF Satcher*, ${ }^{2}$ ER Segura, ${ }^{3}$ A Silva-Santisteban, ${ }^{4,5}$ SL Reisner, ${ }^{6}$ S Sanchez, ${ }^{6}$ JR Lama, ${ }^{2} \mathrm{JL}$ Clark. 'South American Program in HIV Prevention Research (SAPHIR), David Geffen School of Medicine, University of California, Los Angeles, CA, USA; ${ }^{2}$ Program in Global Health, Division of Infectious Diseases, Department of Medicine, David Geffen School of Medicine, University of California, Los Angeles, CA, USA; ${ }^{3}$ Unit of Health, Sexuality and Human Development, Cayetano Heredia University School of Public Health, Lima, Peru; ${ }^{4}$ Department of Epidemiology, Harvard School of Public Health, Boston MA, USA; ${ }^{5}$ The Fenway Institute, Fenway Health, Boston, MA, USA; ${ }^{6}$ Asociación Civil Impacta Salud $Y$ Educación, Lima, Peru

\subsection{6/sextrans-2015-052270.282}

Introduction We explored contexts within which transgender women (TW) and men who have sex with men (MSM) practice insertive anal intercourse (AI) despite receptive role preference (receptive role strain, RRS) and receptive AI despite insertive role preference (insertive role strain, IRS).

Methods We analysed cross-sectional, egocentric data from a 2012-2014 Partner Management study in Lima, Peru. We included MSM/TW reporting an exclusive preference for receptive (pasivo) or insertive (activo) roles and AI with at least 1 of their last 3 non-female partners. Generalised estimating equations with Poisson distribution were used to assess prevalence ratios with 95\% CIs for RRS and IRS during the last sexual encounter, stratified by participant sexual/gender identity. We adjusted for participant pre-sex drug use, condom use, partner type, and partner sexual orientation/role. RRS was assessed for TW and homosexual MSM, and IRS for hetero/bisexual MSM. Results $766 \mathrm{MSM} / \mathrm{TW}$ (median age: 27, IQR: 23-32) provided data for 2,019 recent sexual partners, including: 104 TW with 286 partners, 404 homosexual-identifying MSM with 1,103 partners, and 225 hetero/bisexual-identifying MSM with 554 partners. TW reported the most strain with casual/transactional partners, homosexual MSM with primary/casual partners, and hetero/bisexual MSM with primary partners. TW predominantly (94.2\%) preferred receptive roles and RRS was significantly associated with low education and pre-sex drug use $(\mathrm{p}<0.05$, references: secondary/university, no drug use). Homosexual MSM mainly (75.0\%) preferred receptive roles and experienced RRS more commonly during condomless AI $(\mathrm{p}<0.05$, reference: condom-protected) with partners who preferred receptive/versatile roles $(\mathrm{p}<0.05$; reference: insertive-preferring). Conversely, $77.8 \%$ hetero/bisexual MSM preferred insertive roles and experienced IRS more commonly with insertive/versatile-preferring partners $(\mathrm{p}<0.05)$ than receptive-preferring partners, while less IRS occurred with casual partners ( $<<0.05$; reference: stable).

Conclusion Our findings suggest different MSM/TW vulnerabilities during sexual role negotiation with partners. Future studies/ interventions should consider implications for condom use agency, HIV/STI risk, and sequelae of gender dysphoria.

Disclosure of interest statement None.

\section{P04.29 RELATIONSHIP BETWEEN CIGARETTE SMOKING AND HEALTH RISK BEHAVIOURS AMONG CHINESE YOUTH: FINDINGS FROM A NATIONAL SURVEY}

Wei Guo*. Department of Social Work and Social Policy, School of Social and Behavioral Sciences, Nanjing University, Jiangsu Province, 210023, P. R. China

\subsection{6/sextrans-2015-052270.283}

Introduction Due to their linkages in the social ecology of adolescents, adolescents' cigarette smoking involvement is correlated with other health risk behaviours such as unsafe sexual behaviour and nonsexual risk behaviours, which can damage their health and well-being. This study focuses on examining whether socio-demographic factors and health risk and problem behaviours explain the prevalence of cigarette smoking among Chinese adolescents.

Methods Based on the data from 1st National Youth Reproductive Health Survey in 2009, using binominal and ordered probit models with a Heckman's two-stage estimation procedure, this study examines the socio-demographic and health risk and problem behaviours explaining both cigarette smoking and its frequency, respectively.

Results The results indicate that both the occurrence of cigarette smoking and the frequency of cigarette smoking are mainly associated with health risk and problem behaviours covering other nonsexual risk behaviours and sexual behaviour. The prevalence of smoking was highest among male, rural adolescents, and who had lower education, from one-child family, broken family, or lived in the west. When we adjusted for socio-demographic factors and health risk and problem behaviours, smoking was associated with having premarital sex, having multiple sexual partners, binge drinking, and porn-reading addiction among Chinese adolescent.

Conclusion Unsafe sexual behaviours, binge drinking, and pornreading addiction are correlates of cigarette smoking suggest clustering to form a risk behaviour syndrome among Chinese adolescents. It reflects an urgent need for further exploring the relationship between cigarette smoking and other health risk behaviours will be helpful for designing further tobacco control interventions among Chinese youth. 